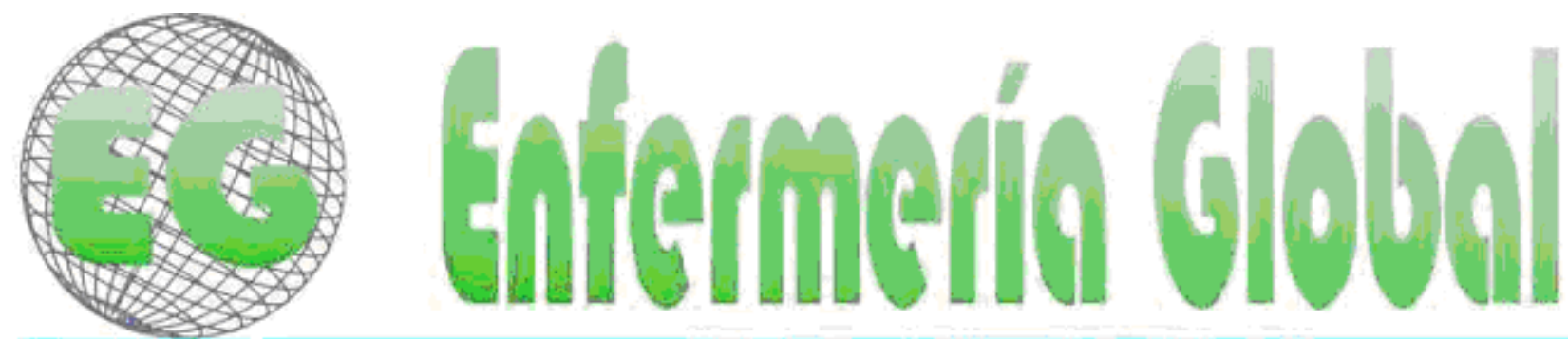

15SN 1696-6*5:

Revista electrônica cuatrimestral de Enfermeria

$\mathrm{N}^{\circ} 20$

Octubre 2010

www.um.es/egloball

REVISIONES

\title{
DIAGNÓSTICOS DE ENFERMERÍA EN PACIENTES INTERNADOS POR QUEMADURAS
}

DIAGNÓSTICOS DE ENFERMAGEM EM PACIENTES INTERNADOS POR QUEIMADURA

\author{
*Barichello , E., "*Silva, MCV., *Barbosa, MH., *Iwamoto, HH. \\ *Doutora em Enfermagem .Professora Adjunta. **Enfermeiro. Universidade Federal do Triângulo Mineiro. \\ Uberaba (MG). Brasil.
}

Palavras-chave: Enfermagem, Hospital, Queimadura.

Palabras clave: Enfermería, Hospital, Quemadura

Keywords: Nursing, Hospital, and Burns

\section{RESUMEN}

El objetivo de este estudio fue localizar, en la literatura brasileña, diagnósticos de enfermería en pacientes de hospital con quemaduras. Se seleccionaron seis artículos para el análisis y fue posible identificar 13 diagnósticos de enfermería a través de una búsqueda bibliográfica en el banco de datos LILACS, de 1998 hasta 2007. Los diagnósticos más comunes fueron dolor y déficit de volumen líquido. Los resultados indican necessidad de más estudios sobre esto tema, para uma buena asistencia de enfermería a esta población.

\section{RESUMO}

O objetivo deste estudo foi identificar na literatura nacional os diagnósticos de enfermagem de pacientes internados, vítimas de lesões por queimaduras. Realizou-se busca bibliográfica no banco de dados LILALCs, entre 1998 e 2007. Foram selecionados seis artigos e identificados 13 diagnósticos de enfermagem. Os diagnósticos de enfermagem prevalentes foram dor e déficit no volume de líquido. Os resultados apontam para a necessidade de ampliar a realização de pesquisas nesta área para subsidiar a assistência de enfermagem desta população

\section{ABSTRACT}

The objective of this study was to identify, in Brazilian literature, nursing diagnoses in hospitalized burn patients. Six articles reporting thirteen nursing diagnoses were found, in a bibliographical search of LILACS database, from 1998 to 2007. The prevailing diagnoses were pain and low body fluid volume. The results indicate a need for further research in this subject, in order to help nursing assistance of this population. 


\section{1- INTRODUCCIÓN}

Actualmente, el coste del tratamiento de pacientes que han sufrido quemaduras es uno de los más caros del sistema de salud y uno de los menos estudiados en cuanto a sus verdaderos costes y resultados. Anualmente, en los Estados Unidos se tratan 1,25 millones de pacientes con quemaduras de los cuales 50.000 precisan ser ingresados. Las quemaduras severas, con tasas de mortalidad del 50\% o más, continúan desafiando a la ciencia. En Brasil, no hay datos estadísticos nacionales sobre el número de pacientes víctimas de quemaduras ni de su mortalidad. Sin embargo, se sabe que siempre suele haber pacientes con quemaduras que se dirigen a los hospitales en busca de asistencia o para ser evaluados. ${ }^{1,2}$

Estar quemado es una de las situaciones más traumáticas que una persona puede vivir física y emocionalmente, pues se trata de algo que interrumpe bruscamente la existencia, pasando de la integridad física al desequilibrio y cuya gravedad varía dependiendo de la extensión de la lesión ${ }^{3}$.

Según la Sociedad Brasileña de Cirugía Dermatológica, las quemaduras son lesiones en los tejidos que envuelven las distintas capas del cuerpo tales como la piel, cabello, pelos, el tejido subcutáneo, músculos, ojos, etc. Generalmente, ocurren debido al contacto directo con objetos calientes como brasas, fuego, llamas, vapores calientes, sólidos sobrecalentados o incandescentes. También pueden ser causadas por sustancias químicas como ácidos y soda cáustica, entre otros. Emanaciones radioactivas como las radiaciones infrarrojas y ultravioletas, o la electricidad, también son factores desencadenantes de las quemaduras. Por lo tanto, las quemaduras pueden tener origen térmico, químico, radioactivo o eléctrico. ${ }^{4}$

Una forma de clasificar las quemaduras es por su profundidad, es decir, por las capas de piel que han sido lastimadas. Así, en cuanto a la profundidad, podemos clasificar este tipo de lesiones como:

a) $1^{\circ}$ grado: afectan las capas superficiales de la piel. Son rojizas, están hinchadas y el dolor local es soportable, no hay ampollas;

b) 2ำ grado: afectan las capas más profundas de la piel. Hay ampollas, la piel está rojiza, manchada o con coloración variable, dolor, hinchazón, desprendimiento de capas de la piel y posible estado de shock;

c) 3ํao: afectan todas las capas de la piel y pueden llegar hasta los huesos. Hay poco o nada de dolor y la piel está blanca o carbonizada. ${ }^{5}$

El daño al inicio de una quemadura y el consiguiente tratamiento pueden agotar a las personas mucho más allá de su nivel de tolerancia. El quemado tiene que luchar contra la molestia física inicial y con el dolor, además de los problemas psicológicos inmediatos y tardíos, como delirios, depresión y de su imagen corporal alterada. La asistencia dispensada a los quemados requiere del profesional una formación técnica, pues el cuidado clínico de enfermería es amplio y se extiende de la fase clínica aguda de la herida quemada hasta la fase de rehabilitación. ${ }^{6}$

En esa secuencia, el enfermero debe estar preparado para atender al paciente quemado porque éste presenta mucho sufrimiento físico y, consecuentemente, psicológico, debido a las secuelas resultantes y/o de las marcas severas dejadas en su cuerpo. ${ }^{3}$ 
La sistematización de la asistencia de enfermería (SAE) implica el desempeño de la práctica por los profesionales de enfermería de forma sistemática mediante una actividad deliberada, lógica y racional; el poseer un amplio conocimiento es esencial para valorar el estado de salud del paciente, emitir juicios, diagnósticos, efectuar planificación y valoración de las acciones de enfermería de forma apropiada. Se considera que la SAE incluye cinco pasos interrelacionados: recogida de datos, diagnóstico de enfermería, planificación, implementación y valoración. ${ }^{3,7}$

La SAE es un recurso que le permite al enfermero organizarse y planificar los cuidados adecuados a los pacientes. De esta forma, contribuye a consolidar esta profesión y su autonomía. ${ }^{3,7}$

El enfermero de hoy debe comprometerse incesantemente con la adquisición de nuevos conocimientos de modo a actuar de forma efectiva en el equipo de salud con miras a mejorar las condiciones de vida de la clientela que atiende. La práctica, determinante de la posición del enfermero en la sociedad, asegura beneficios simbólicos que le permiten mantener su autonomía en el espacio social. ${ }^{8}$

El Diagnóstico de Enfermería (DE) se torna un vehículo adecuado para articular la enfermería a las demás profesiones de la salud. Así, es factible emplear una taxonomía única con aplicabilidad en sistemas informatizados para transmitir la práctica y definir las perspectivas de la profesión en cuanto al cuidado. La pretensión de este sistema de apoyo a la decisión es agilizar, estandarizar y concretar la práctica de la sistematización de la asistencia de enfermería empleando los diagnósticos de enfermería según la taxonomía de la North American Nursing Diagnosis Association - NANDA. ${ }^{9}$

Siendo así, los enfermeros deben poder contar con oportunidades que hagan viable la ampliación y actualización de los conocimientos científicos, que sirvan tanto para incorporar capital cultural como también para poder ser críticos en cuanto a la práctica asistencial.

En este sentido, el presente trabajo busca exponer los diagnósticos de enfermería encontrados en la literatura nacional, elaborados durante la hospitalización de pacientes que sufrieron quemaduras.

\section{2- OBJETIVO}

- Identificar en la literatura nacional los diagnósticos de enfermería en los pacientes ingresados, víctimas de lesiones por quemaduras.

\section{3- MÉTODO}

Esta investigación científica es un estudio de revisión de la literatura. ${ }^{10}$

El relevamiento bibliográfico estuvo delimitado por artículos publicados en portugués entre 1998 y 2007, que enfocaban diagnósticos de enfermería en pacientes quemados e ingresados.

Se realizó una búsqueda bibliográfica en el tesauros de la Literatura Latinoamericana en Ciencias de la Salud (LILACs), habiéndose utilizado las siguientes agrupaciones de palabras clave: "enfermería y quemaduras y diagnóstico"; "quemaduras y hospital y enfermería"; "quemaduras y alta hospitalaria"; "quemaduras y planificación". 
Fueron identificados nueve artículos que enfocaban este tema y luego de su lectura y análisis, fueron seleccionados seis artículos que atendían a los criterios de inclusión del presente estudio.

La búsqueda por el tesauro de tesis y disertaciones de la Universidad de Sao Paulo tuvo como palabra clave "quemadura". En este tesauro fueron identificadas trece disertaciones y tesis, seleccionándose sólo una que, después de su lectura y análisis, no fue incluida en este estudio por no atender a los criterios de inclusión establecidos previamente.

\section{4- RESULTADOS Y ANÁLISiS}

Después de la lectura de los nueve artículos identificados por la búsqueda bibliográfica, seis publicaciones atendían a los criterios de inclusión de esta investigación. A pesar de que para este estudio el período de tiempo de las publicaciones era de 10 años, de 1998 a 2007, se puede percibir que el tema aún no tiene gran impacto en la literatura nacional.

A continuación, en el cuadro 1 se muestra la literatura científica identificada sobre el tema de esta investigación, según los autores, tipo de investigación realizada, objetivos y resultados encontrados. 
Quadro 1 - Autores, tipos de estudo, objetivose os resultados identificados nos artigos. Uberaba (2007).

\begin{tabular}{|c|c|c|c|}
\hline Autores & Tho de estrido & Objetivos & Resultados \\
\hline $\begin{array}{l}\text { Carhuci } \\
\text { VDS, } \\
\text { et.sl }\end{array}$ & $\begin{array}{l}\text { Descritivo } \\
\text { retrospectiso }\end{array}$ & 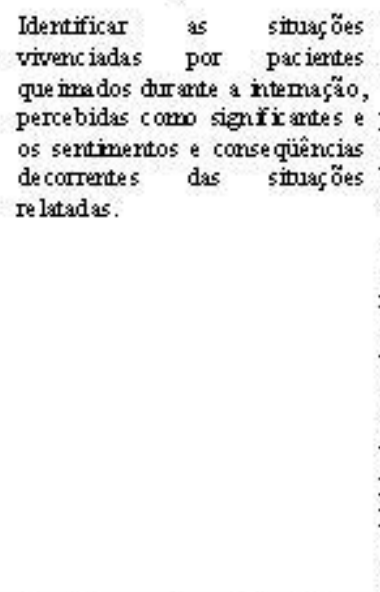 & 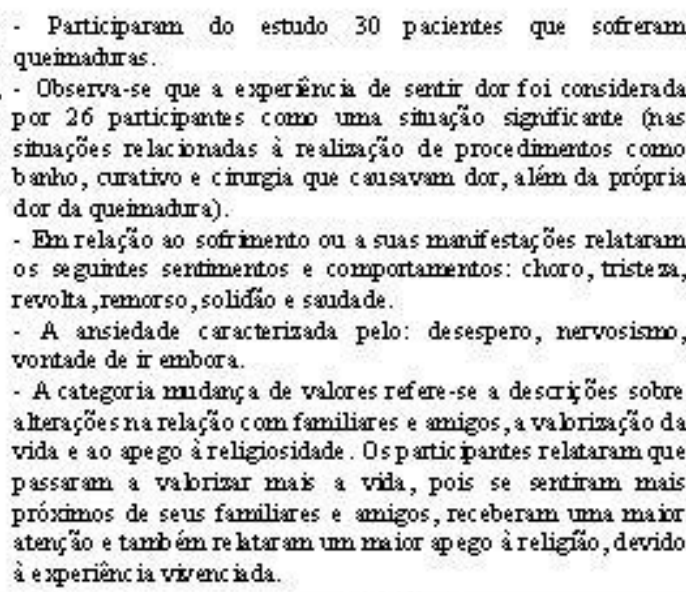 \\
\hline $\begin{array}{l}\text { Mene ghett } \\
\text { i RAS, } \\
\text { et.al. }\end{array}$ & Retrospectivo & $\begin{array}{l}\text { Idertificar os diagrósticos de } \\
\text { enfermagem de pacientes } \\
\text { adultos, } \\
\text { admitidos em una Unidade de } \\
\text { Que imados. }\end{array}$ & 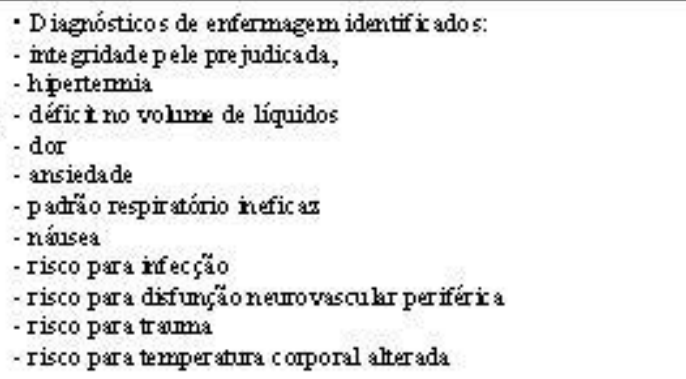 \\
\hline $\begin{array}{l}\text { Focha } \\
\mathrm{RD}, \\
\text { Freitis A }\end{array}$ & Descritivio & $\begin{array}{l}\text { Construir um protocolo de } \\
\text { auilado pars os pacientes com } \\
\text { que imadirss. }\end{array}$ & 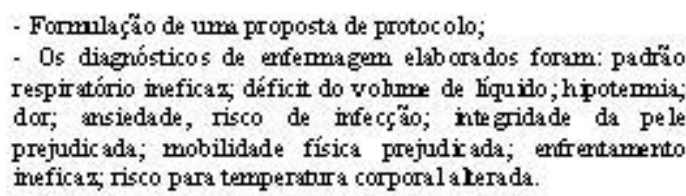 \\
\hline $\begin{array}{l}\text { Rossi LA, } \\
\text { et.sl }\end{array}$ & Enográfico & 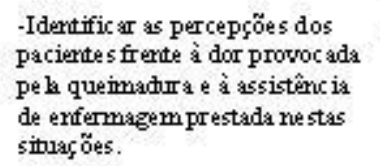 & - Dor re hacionado as proce dimentos de barkho e curativo. \\
\hline $\begin{array}{l}\text { Fossi LA, } \\
\text { et. al }\end{array}$ & $\begin{array}{l}\text { Descritivo } \\
\text { Prospectivo }\end{array}$ & $\begin{array}{l}\text { Descrever o perfil das } \\
\text { caracter ític as def nitioras do } \\
\text { paciente com diagróstio o de } \\
\text { enfermagem Déficit de vohme } \\
\text { de líquidos. }\end{array}$ & Déficit de vohnme de líquidos. \\
\hline $\begin{array}{l}\text { Scherer } \\
\text { ZAP, Luis } \\
\text { MAV }\end{array}$ & Qualitativo & $\begin{array}{l}\text { Conthe cer o significado de estar } \\
\text { que ima do experimentado pe lo } \\
\text { paciente durante o periodo da } \\
\text { internas, ão. }\end{array}$ & 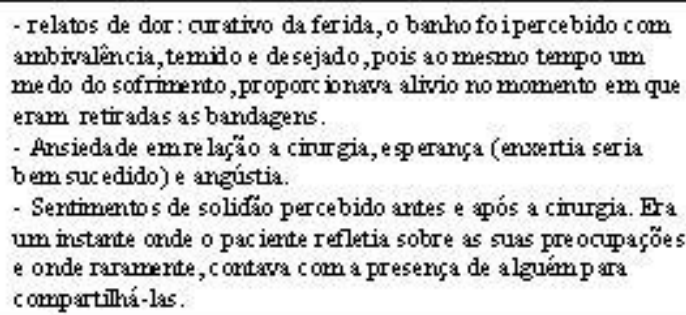 \\
\hline
\end{tabular}

En el cuadro 1 se observa que entre los estudios identificados en esta investigación, cuatro $(66,66 \%)$ son de enfoque cuantitativo y delineamiento no experimental y dos $(33,33 \%)$ son cualitativos. Además, se observa que los estudios cualitativos no incluían los diagnósticos de enfermería definidos y que algunos diagnósticos pueden definirse un poco más por el relato de los profesionales y pacientes.

En el cuadro 2 se presentan los diagnósticos de enfermería identificados en las publicaciones, los factores relacionados y las características definidoras. 
Quadro 2 - Diagnósticos de enferm agem segundo NANDA, caraderísticas de finidoras e fatores relacionados identificados nos artigos. (Uberaba, 2007).

\begin{tabular}{|c|c|c|}
\hline Diagnósticos de Enfermagem & Caraderísticas definidoras & Artigos \\
\hline Ansiedade & Ansioso; inquietaçẫo; confusẫo; náusea; tontura; medo. & 3 e 7 \\
\hline Déficit de volume de líquido & $\begin{array}{l}\text { Perda ativa de volume de líquidos; perda de líquidos por vas } \\
\text { anorm ais; diminuição do débito urinário; enchimento venoso } \\
\text { dim inuído; turgor diminuído. }\end{array}$ & $3,7,12$ \\
\hline $\begin{array}{l}\text { Desobstrução Ineficaz das vias } \\
\text { aéreas }\end{array}$ & Edern a; dispnéia; treqüência respiratória aum entada; dor & 3 e 7 \\
\hline Dor & $\begin{array}{l}\text { Queixoso; choroso; gemente; pouco comunicativo; relato } \\
\text { verbal; mudança do apetite; distúrbio do sono; lesấo de } \\
\text { tecido e nervos }\end{array}$ & 3,6 e 7 \\
\hline Enfrentamento ineficaz & Medo e ansiedade & 7 \\
\hline Integridade da pele prejudicada & $\begin{array}{l}\text { Destruiçấo de camadas da pele; estados dos líquidos } \\
\text { alterados; estado metabólico alterado }\end{array}$ & 3 e 7 \\
\hline Mobilidade física prejudicada & Edern a; dor; desconforto; contraturas m usculares & 3 \\
\hline $\begin{array}{l}\text { Risco para disfunçẫo neuro } \\
\text { vascular }\end{array}$ & "Trauma; obstruçã̃o vascular & 3 \\
\hline Risco para in fecçẫo & $\begin{array}{l}\text { *Trauma; defesas prim ária insuficientes; procedimentos } \\
\text { invasivos; exposiçẫo am biental aumentada }\end{array}$ & 3 \\
\hline $\begin{array}{l}\text { Risco para tem peratura corporal } \\
\text { alterada }\end{array}$ & "Trauma; taxa metabólica alterada; desidrataçẫo. & 3 e 7 \\
\hline Risco para trauma & "Dificuldades no equilíbrio; traum a prévio. & 3 \\
\hline
\end{tabular}

Se observa que el diagnóstico de enfermería de dolor y déficit de volumen de líquidos fueron los más citados entre los estudios analizados. Se sabe que, en la mayoría de los casos, los pacientes con quemaduras sienten intenso dolor y que precisan ser sedados cuando la lesión ocupa grandes superficies. El déficit de volumen de líquidos es una de las inquietudes inmediatas en las quemaduras. Los pacientes portadores de quemaduras sufren una gran pérdida de fluidos porque el plasma traspasa del compartimiento intravascular al espacio intersticial. Esta pérdida es proporcional a la extensión y profundidad de la lesión. La ansiedad y la integridad de la piel perjudicada aparecen en dos artículos y en otros artículos, aunque no hayan sido clasificados como diagnósticos de enfermería, se mencionan como una situación enfrentada por el paciente. ${ }^{11}$

En el cuadro 3 se muestran los artículos que no incluían los diagnósticos de enfermería propiamente dichos. Con los factores relevados por los autores se lograron detectar características definidoras y factores relacionados y elaborar, entonces, los diagnósticos de enfermería. 
Quadro 3- Diagnósticos de enfermagem elaboradas a partir dos fatores apontados na literatura

(Uberaba, 2007).

\begin{tabular}{|c|c|c|c|}
\hline Artigos & $\begin{array}{l}\text { F atores apontados pelos autores para } \\
\text { os diagnósticos }\end{array}$ & $\begin{array}{c}\text { Caraderísticas definidoras da } \\
\text { NANDA }\end{array}$ & Diagnóstico \\
\hline 11 e 13 & $\begin{array}{l}\text { Desespero; nervosismo; wontade de ir } \\
\text { embora; preocupaçẫo em relaçẫo a } \\
\text { dinurgia; esperança (enxertia seria } \\
\text { bern sucedido); angústia. }\end{array}$ & $\begin{array}{l}\text { Agit açẫo; angústia; nervoso; } \\
\text { preocupaçốes expressas. }\end{array}$ & Ansiedade \\
\hline 11 & $\begin{array}{l}\text { Valorizaçã̃o da vida; apego à } \\
\text { religiosidade; proximidade de } \\
\text { familiares e amigos. }\end{array}$ & $\begin{array}{l}\text { Força unificada; Fonte } \\
\text { sagrada; Sentimentos de } \\
\text { consciência. }\end{array}$ & $\begin{array}{l}\text { Disposiçẫo para bern - } \\
\text { estar espiritual } \\
\text { aumentado }\end{array}$ \\
\hline 11 e 13 & $\begin{array}{l}\text { Realizaçẫo de procedimentos como: } \\
\text { retirada das bandagens, nas } \\
\text { situaçốes relacionadas à banho, } \\
\text { durativo, além da própria dor da } \\
\text { queimadura. }\end{array}$ & $\begin{array}{l}\text { Relato verbal; gestos } \\
\text { protetores; foco em si próprio; } \\
\text { agenteslesivos (físicos) }\end{array}$ & Dor \\
\hline 11 & $\begin{array}{l}\text { Relatos dos seguintes sentim entos e } \\
\text { com portamentos choro, tristeza, } \\
\text { revolta, rem orso, solidẫo e saudade. }\end{array}$ & $\begin{array}{l}\text { Baixo estado de otimismo; } \\
\text { estado emocional intenso; } \\
\text { estressores múltiplos. }\end{array}$ & Adaptaçẫo prejudicada \\
\hline
\end{tabular}

A pesar de que el dolor y la ansiedad no hayan sido descritos como diagnósticos de enfermería en estos estudios, al seleccionar los factores señalados por los autores tales diagnósticos pueden ser evidenciados.

\section{5- CONSIDERACIONES FINALES}

El presente estudio es sumamente importante para orientar la asistencia de enfermería a pacientes quemados, teniendo en cuenta que son pocos los centros de referencia para el cuidado de dichos pacientes.

Corresponde realzar la escasez de trabajos científicos en la literatura nacional llevados a cabo por profesionales de enfermería, principalmente en relación a la sistematización de la asistencia de enfermería.

Los diagnósticos de enfermería prevalentes a partir de los estudios analizados fueron dolor y déficit de volumen de líquidos y ansiedad, lo que señala la necesidad de que el profesional tenga en cuenta no sólo los problemas fisiopatológicos, sino también los trastornos psicosociales en tales pacientes.

Estos hallazgos podrán orientar la asistencia de enfermería a pacientes quemados, principalmente donde no haya centros de referencia para tratamiento en esta especialidad.

Considerando la relevancia del tema y observando la escasez de artículos nacionales sobre este asunto, uno de los focos para garantizar que mejore la asistencia a dichos pacientes es fomentando nuevas investigaciones en este campo de la salud. 


\section{REFERENCIAS BIBLIOGRÁFICAS}

(1) Sanchez JLA, Pereperez SB, Bastida JL, Martinez MM. Cost-Utility Analysis Applied to the Treatment of Burn Patients in a Specialized Center. Arch Surg 2007; 142: 50-57.

(2) Torratia FG, Rossi LA, Ferreira E, Dalrid CB, Carvalho EC, Barbeira CB, Santos. Analysis of cost of dressings in the care of burn patients. Burns 2000; 26: 289- 293.

(3) Meneghetti RAS, Rossi LA, Barruffini RCP, Dalri MCB, Ferreira E. Planejamento da assistência a pacientes vítimas de queimaduras: relação entre os problemas registrados e cuidados prescritos. Rev Esc Enferm USP 2005; 39 (3): 268-279.

(4) Sociedade Brasileira de Cirurgia Dermatológica. Queimaduras: orientações e cuidado. 2004 Fev. [acesso em 2007 jun. 12]. Disponível em:

http://www.sbcd.org.br/pagina.php?id=75.

(5) Brasil. Ministério da Saúde. Dicas em saúde e queimadura. 2004 maio. [acesso em 2007 out. 25]. Disponível em: http://bvsms.saude.gov.br/html/pt/dicas/54queimaduras.html

(6) Rossi LA, Camargo C, Santos CMNM, Barruffin RCDP, Carvalho EC. A dor da queimadura: terrível para quem sente, estressante para quem cuida. Rev.latinoam.enfermagem 2000; 8 (3): 18-26.

(7) Rocha RD, Freitas A. Unidade de tratamento de queimados: relato de experiência sobre a construção de protocolo para os cuidados de enfermagem. Rev elet sem enf 2003; (3): 111

(8) Bittar DB. Sistematização da assistência de enfermagem ao paciente crítico: proposta de um instrumento de coleta de dados. [trabalho de conclusão de curso]. Uberaba: Centro de Graduação em Enfermagem, Universidade Federal do Triangulo Mineiro, 2005.

(9) North American Nursing Diagnosis Association. Diagnósticos de Enfermagem da NANDA: definições e classificação 1999-2000. Porto Alegre: Artmed; 2004.

(10) Polit, DF, Beck, CT; Hungler, BP. Fundamentos de pesquisa em enfermagem: métodos, avaliação e utilização. 5aㅡ ed. Porto Alegre: Artes Médicas; 2004.

(11) Rossi LA, Dalri MCB, Ferraz AEP, Carvalho ECD, Hayashida M. Déficit de volume de líquidos: perfil de características definidoras no paciente portador de queimadura. Rev.latinoam.enfermagem 1998; 6 (3): 85-94. 